\title{
LES INTERFÉRENCES DU CHANTIER DE RECONSTRUCTION DANS L'ORGANISATION DE LA VIE MONASTIQUE
}

\section{NICOLAS REVEYRON}

UDC: $726.71: 69$ $27-788$

Review

Manuscript received: 17. 12. 2016.

Revised manuscript accepted: 07. 02. 2017.

DOI: 10.1484/J.HAM.5.113732

\author{
N. Reveyron \\ Université Lyon 2 \\ RAR / UMR 5138 " Archéologie et Archéométrie » \\ Maison de l'Orient et de la Méditerranée 7 rue Raulin \\ 69365 Lyon cedex 7, France \\ nicolas.reveyron@univ-lyonz.fr
}

Maintenance, enhancement or reconstruction, performed inside a monastery in any time period negatively affected the monastic life. This challenges equally impact males and females (both gender). Of course, nuns are more affected by the interventions of workers, whether lay or monastic inside the enclosure. Even if the textual sources are not explicit, archaeology reveals different strategies to approach these challenges.

Keywords: Cluny, Nantua, de Paray-le-Monial, Saint-Germain d'Auxerre, Vézelay, Saint-Gilles-du-Gard, Saint-Jean d'Arles, Berthe, Girard, église mariale, chantier, réparation, reconstruction, ouvriers laïcs, pèlerin, chantier allotopique, chantier hétérotopique, chantier homotopique

Les travaux de construction, d'entretien ou d'aménagement constituent un aspect important de la vie monastique. L'assertion n'est paradoxale qu'en apparence. Les traditionnelles divisions académiques du savoir ont en effet rangé la question du chantier dans les champs du profane, des techniques ou de la sociologie, et les problématiques «monastiques», dans le domaine du religieux (on dirait aujourd'hui: du «fait religieux»). Qui plus est, les monastères légués par le Moyen Age, devenus souvent des monuments historiques et restaurés dans le respect de l'état supposé d'origine, nous les percevons comme des ensembles architecturaux définitivement stables, vides d'occupants et figés dans l'évocation d'une vie spirituelle ancienne qui trouve son expression le plus émouvante dans l'église, avec ou sans stalle, et dans la sonorisation en grégorien pouvant accompagner la visite; à peine entraperçoit-on d'ailleurs quelque chose d'un quotidien plus trivial dans la cuisine ou le réfectoire, moins encore dans le dortoir dont l'ampleur architecturale impressionne les visiteurs et l'absence de mobilier gomme l'identité objective.

Toutefois, les progrès faits au siècle dernier dans des aspects très concrets des institutions monastiques - rôle économique, politique, technologique... - ont permis de recentrer les questionnements sur les réalités matérielles de la vie monastique. Ainsi, en 1964, Anselme Dimier publiait chez Fayard une belle synthèse sur la vie monastique au Moyen Age, ayant pour titre: Les moines bâtisseurs, et sous-titre: Architecture et vie monastique, mais le projet était plutôt orienté sur les rapports entre les compositions architecturales et les formes de vie cénobitique ou semi-érémitique. Parallèlement, les recherches sur la construction médiévale se sont focalisées depuis le XIX ${ }^{\mathrm{e}}$ siècle soit sur l'architecture de défense, soit, de façon plus emblématique, sur les chantiers des cathédrales ${ }^{1}$, sous-entendu des cathé- drales gothiques, considérées comme le parangon de l'art de bâtir et les meilleurs exemples du progrès des techniques au Moyen Age. Aujourd'hui, la question est abordée sous d'autres angles et dans d'autres orientations euristiques ${ }^{2}$. Les avancées accomplies par l'archéologie, et singulièrement par l'archéologie du bâti, permettent de considérer le même sujet, mais dans une visée dynamique: quelles ont été au Moyen Age les incidences d'un chantier de construction au sein d'un monastère, dans une situation qui mêlait donc l'ancien et le nouveau, le travail de force et la prière communautaire?

\section{LA PLACE DES TRAVAUX DANS L'ORGANISATION DE L'ESPACE MONASTIQUE}

Dans le monde monastique, la construction, la transformation ou simplement l'entretien d'édifices formant le carré du cloître occasionnent des perturbations lourdes de la vie commune. D'une part, en effet, les campagnes de travaux lourds introduisent au sein du monastère des laïcs, étrangers à la vie religieuse et inadaptés aux rigueurs de la règle, ou bien bousculent les habitudes, quand il s'agit de moines travaillant eux-mêmes sur le chantier. D'autre part, les reconstructions font disparaître temporairement ou mettent momentanément hors d'usage des bâtiments attachés d'une manière ou d'une autre aux activités des moines.

\section{1-Les hommes du chantier}

Toutes ces opérations posent des problèmes concrets de cohabitation entre les hommes du chantier, laïcs ou moines, et les membres du convent. Le terme d'ouvrier - operarius, le plus fréquemment employé, est relativement imprécis. Il désigne aussi bien celui qui travaille de ses mains à la

\footnotetext{
${ }^{1}$ Voir par exemple J. GIMPEL, Les bâtisseurs de cathédrales, Paris, 1958.

${ }^{2}$ Pour une réflexion récente sur le sujet, voir M.C. Somma (a cura di), Cantiere e maestranze nell'Italia medievale, Atti del convegno (Chieti-San Salvo, 16-18 mai 2008), Spoleto, 2010 (De re monastica, II).
} 
construction que celui qui supervise les travaux ou dirige le chantier, voire les fabriciens. Mais la question de la cohabitation est peu abordée dans les textes normatifs. Ainsi, la règle de saint Benoît reste muette au sujet des laïcs œuvrant dans le monastère. Et quand elle traite des moines artisans, elle parle uniquement, et sous un aspect moral, de ceux qui produisent des biens de consommation. Dès le Moyen Age central, pourtant, certains ordres ont acquis une réputation de constructeurs. La référence est bien connue: «Tous les monastères des Cisterciens - écrit Orderic Vital au XII ${ }^{e}$ siècle -, sont construits dans le désert et au milieu des bois, et ces religieux les bâtissent de leurs propres mains». Et même s'il faut faire la part d'une certaine exaltation ${ }^{3}$, on doit reconnaître que la question relève pleinement du domaine de l'histoire de l'art. De fait, les activités des moines dans le domaine de la construction apparaissent très diverses.

En 1961, M. Aubert notait déjà que «comme au temps des grandes fondations mérovingiennes et carolingiennes, les abbayes des $\mathrm{XI}^{\mathrm{e}}$ et $\mathrm{XII}{ }^{\mathrm{e}}$ siècles étaient des centres d'art. Il y avait parmi les moines des maçons, des tailleurs de pierre, des charpentiers capables de diriger non seulement les ouvriers de leur spécialité, mais l'ensemble du chantier [...]. Les abbayes bénédictines et clunisiennes étaient plus libérales et nombreux sont les exemples de moines, architectes, sculpteurs ou peintres prêtés par leur abbaye à des maisons d'un autre ordre ou même à des évêques pour les aider dans leurs travaux» ${ }^{4}$. Chroniques et vitae font état de la participation de moines spécialistes à des chantiers religieux. Au Mans, par exemple, c'est à un moine-maçon de la Trinité de Vendôme, Jean, qu'Hildebert de Lavardin confie la reconstruction de la nef de la cathédrale, consacrée en 1120. A Saint-Front-de-Périgueux, le chanoine Itier fait appel à un moine de La Chaise-Dieu, Guinamond, pour sculpter le tombeau du saint, tandis qu'à Saint-Benoît-sur-Loire, Gauzlin fait venir de l'abbaye de Saint-Julien de Tours un moine-peintre nommé Odolric ( $c f$. infra) .

Il est vrai que les sources mentionnent plus volontiers les moines artistes que les moines maçons - souvent d'ailleurs directeurs des travaux plutôt qu'ouvriers -, transmettant en cela une image fidèle des hiérarchies médiévales. Pour saint François d'Assise, par exemple, «Celui qui travaille avec ses mains est un ouvrier. Celui travaille avec ses mains et sa tête est un artisan. Celui travaille avec ses main, sa tête et son cœur est un artiste». Et les travaux le plus durs apparaissent aussi le plus dégradants: «A Dieu ne plaise - explique Orderic Vital -, que de vaillants chevaliers, des philosophes subtils, d'éloquents docteurs, parce qu'ils ont renoncé au siècle, soient obligés, comme de vils esclaves, à s'appliquer à des travaux pénibles et peu convenables» ${ }^{6}$. L'archéologie du bâti est susceptible d'apporter des éléments de réponse, notamment dans l'analyse des traces d'outil et l'étude des marques lapidaires laissées comme des signatures par les tailleurs de pierres sur les parements des bâtiments. Il y a là un vaste gisement d'études à venir, mais on remarque déjà que dans certains monastères, notamment de l'ordre de Citeaux, les blocs sont nombreux à porter des marques qu'il faut attribuer à des tailleurs de pierre sans doute laïcs, les moines n'ayant pas la nécessité d'inscrire ainsi leur travail dans un système de rétribution.

\section{2-Des laïcs dans la clôture}

L'intervention d'ouvriers laïcs à l'intérieur des monastères apparaît ponctuellement dans diverses sources écrites, des chartes et des contrats aux chroniques et aux vitae; on y rencontre même parfois des scènes très vivantes comme en 1169, dans l'abbaye de Saint-Trond, l'abbé Wiricus qui presse incessamment (jugiter: «comme un écoulement continu») les ouvriers pour respecter les délais: «Igitur cum abbas Wiricus opus ceptum festinato consummari vellet, et ad id peragendum operarios jugiter urgeret, tandem expleto triennio et amplius Deo auspice totum pro voto consummavit» ${ }^{7}$. Dans ce domaine, la littérature romanesque aussi s'avère d'un apport non négligeable, parce qu'elle convoque volontiers des détails réalistes et des situations que les autres sources négligent. La Chanson de Girard de Roussillon fait ainsi la part belle au chantier religieux ${ }^{8}$. Mais l'histoire du comte Girart, on le sait, c'est d'abord la fondation et la construction de l'abbaye de Vézelay ${ }^{9}$, près de laquelle, après la défaite du comte, Berthe, sa femme, trouve refuge. Avec l'aide d'un pèlerin, elle effectue, de nuit et jusquà l'épuisement de ses forces, un travail pénitentiel sur le chantier de l'abbaye ${ }^{10}$. Outre leurs activités de transport de pierres, de mortier ou d'eau, ils remplissent ensemble des sacs de sable qu'ils montent à deux depuis la carrière jusque sur la colline ${ }^{11}$. Girart, en fuite, surprend ce manège, où son

\footnotetext{
${ }^{3}$ Le thème a nourri le très beau roman de Fernand Pouillon, qui s'inscrit dans ce mouvement de retour à la réalité concrète de la construction comme norme de l'esthétique architecturale, illustré notamment par les recherches sur l'architecture monastique du Moyen Age. F. POUILLON, Les pierres sauvages, Paris, 1964.

${ }^{4}$ M. AUBERT, La construction au Moyen Age, in Bulletin Monumental, 118, 4, 1961, p. 17.

${ }^{5}$ V. MORTET, Recueil de textes relatifs à l'histoire de l'architecture et à la condition des architectes au moyen-âge (XIİ̀me-XIII ${ }^{\text {ème }}$ siècle), Paris, 1911, p. 242243 et 36 .

${ }^{6}$ Ibidem, p. 17-18.

${ }^{7}$ Gesta abbatum sancti Trudonis, gestorum abbatum sancti Trudonis continuatio secunda, LIV, cap. 5. V. MORTET, P. DESCHAMPS, Recueil de textes relatifs à l'histoire de l'architecture et à la condition des architectes au moyen-âge (XII ${ }^{\text {ème-XIII }}{ }^{\text {èe }}$ siècle), Paris, 1929, p. 12.

${ }^{8}$ Elle évoque par exemple les tailleurs de pierre juste après la bataille de Verdonnet, lorsqu'il est fait mention des salaires des ouvriers qui façonnent les sarcophages pour les chevaliers tués. Laisse 345, v. 5354-5362: «Li bib e li abat, nostre doutor, Facem un cimenterre en Deu onor. Icil qui ci sunt mort, li nostre el lor, Cascuns i est restaz por son seinor. Ere l'ont otreiat tuit li meillor. Mil mars en deit abbes a son prior Senz ço dunt sunt loiat li taillador; E pois lor sert tostens de tal labor Tan com furent en trez gerreedor». La chanson de Girart de Roussillon, Traduction présentation et notes M. De Combarieu et G. Gouiran, Paris, 1993, p. 408-409. Pour un commentaire historique, voir C. TREFFORT, Des sarcophages réels aux légendes épiques: réflexion autour d'une archéologie littéraire, in De l'écrin au cercueil: essais sur les contenants au Moyen Age, dir. D. James-Raoul et C. Thomasset, Paris, 2007, p. 93-116.

9 R. LOUIS, De l'histoire à la légende, Girart, comte de Vienne (...819-877), et ses fondations monastiques, Auxerre, 1946.

${ }^{10}$ La chanson de Girart de Roussillon, op. cit. (n. 8), laisses DCXLVII-DCXLIX.

${ }^{1 "}$ «Porte peire u morter u aige a tine [...] Eu voil portar od tei, al ne demant, Aige, peire u sablon, u pauc u grant [...] Ere ferant issi cum l'unt enpres: Aportent en sablon d'aval u es Sobr'un tinal en sac, quant l'i ont mes». Ibidem, v. 9564, v. 9580-81 et v. 9590-92.
} 
épouse donne l'impression de s'humilier devant un homme: «Le comte voit le pélerin remplir de sable le sac que Berthe agenouillée lui tend [...]. Il voit sa femme Berthe qui tend le sac; lui y met le sable qu'il tire du sol ${{ }^{12}}^{2}$. Un compagnon du comte tente de lui expliquer la situation, en racontant que lors de la construction de Saint-Sophie de Constantinople, l'empereur, par souci de sa gloire personnelle, avait interdit aux laïcs de participer au chantier, mais qu'une pauvre femme, par pénitence, s'était attachée à nourrir les bêtes du chantier et à transporter de l'eau pour préparer le mortier ${ }^{13}$. Ce texte remarquable donne un éclairage cru sur la sociologie du chantier religieux et la multiplicité des petites activités assurant la bonne marche des travaux.

Si la présence de la comtesse et du pèlerin sur le chantier de Vézelay renvoie à une problématique religieuse - un travail rédempteur mis au service de l'église -, à l'inverse, l'intervention des laïcs du chantier dans l'église même pose un problème de cohabitation: la sacralité du lieu et des actions liturgiques tend à les en exclure. La question s'est posée dans toute sa complexité lors de la reconstruction de l'abbatiale de Saint-Germain d'Auxerre sous l'abbé Jean (1252-1278). La démolition des parties vétustes qui a précédé le début des travaux a amené la découverte de reliques dans les autels, mais la liturgie qui devait accompagner leur translation ne pouvait pas être célébrée devant des ouvriers laïcs («minus digne minusque reverenter inter laïcos operarios tractaretur») $)^{14}$. Cependant, la valeur reconnue à certains artistes aplanit bien des difficultés, comme le montre le cas de Foulque, homme de condition servile dépendant de Saint-Aubin d'Angers à la fin du XI ${ }^{e}$ siècle. Mandé à l'abbaye pour des travaux de peinture et de vitraux, il comparaît au chapitre devant l'abbé et les moines réunis à cette occasion, devient convers («Et ibi frater eorum devenit»), puis est affranchi par l'abbé Girard. La charte stipule qu'il recevra en contrepartie de son travail une maison et un arpent de vignes dont hériterait son fils, le cas échéant ${ }^{15}$.

Des documents juridiques ou comptables comme le Contrat de louage passé [en 1261] entre l'abbé de SaintGilles-du-Gard et l'intendant des travaux de l'abbaye d'une part, de l'autre, maître Martin de Lonay pour l'achèvement de l'église, publié par Victor Mortet et Paul Deschamps en 1929, couvrent un champ d'étude beaucoup plus restreint, mais apportent d'utiles précisions sur l'adaptation du laïc aux coutumes monastiques: «Si vero idem magister Martinus voluerit infra monasterium comedere diebus carnalibus, scilicet die dominica, die lune, die martis, et die jovis, liceat ei et possit comedere in hospicio et in mensa dicti domini abbatis vel sociorum ejus, si dominus abbas [afuerit]; sin autem, cum judice vel cum decano vel cum eis qui tunc ibi comedent. Et si voluerit dictis diebus carnalibus comedere ad dictam mensam vel in hospitio domini abbatis, dictus operarius, vel qui pro tempore fuerit, teneatur ei dare et providere in porcione unius monachi, scilicet in tribus denariis Turonensium; et in ceteris diebus non carnalibus et jejunalibus habebit generale et pitanciam unius monachi, et illud generale et illam pitanciam accipiet inferius in coquina $»^{16}$.

Ce document est très précieux en ce qu'il permet de comprendre par quels biais - au milieu du XIII ${ }^{e}$ siècle et dans l'espace languedocien - l'intervention d'artisans laïcs a pu être intégrée au temps du monastère, pendant la durée des travaux. Le principe premier qui régit ces relations pour ainsi dire oxymoriques est de conformer le rythme de la vie laïque à celui de la vie des moines, comme si le laïc devenait momentanément moine, ou plutôt convers. D’ailleurs, pour ce qui est de la nourriture, la quantité de référence est la portion attribuée au moine, alors qu'on attendrait un repas plus conséquent pour un travailleur de force, en temps normal et, plus encore, en période de jeûne. Le texte développe à ce propos une casuistique complexe, fondée sur des attendus très strictes. Il distingue tout d'abord les diebus carnalibus et les diebus non carnalibus et jejunalibus, critères réguliers à partir desquels sont définis d'une part, la quantité et la qualité de la nourriture servie à l'artisan (in porcione unius monachi, scilicet in tribus denariis Turonensium, dans le premier cas; pitanciam unius monachi, dans le second), d'autre part le lieu où l'artisan peut les consommer, soit à la table de l'abbé (ou d'un de ses représentants, répartis en deux groupes selon le degré de familiarité) dans le premier cas, soit à la cuisine, dans le second. La précision du vocabulaire (l'opposition entre porcione et pitanciam, par exemple) et des données concrètes (désignation d'abord typologique, puis nominative des diebus carnalibus) est révélatrice: le texte se présente formellement comme une sorte d'avenant à la règle, rédigé et codifié comme tel. Dans ces conditions, la mention d'hospitio oblige à nuancer la position de l'artisan: il est aussi l'hôte de l'abbé, position privilégiée justifiant sa présence à la table abbatiale. Traité en hôte, comme tout laïc établissant une relation de réception temporaire avec le convent, et revêtu d'une apparence monastique, qui lui ouvre l'intérieur du monastère, maître Martin peut ainsi avoir accès à l'église et aux édifices majeurs, c'est-à-dire le lieu où la règle prime sur toute autre chose.

\footnotetext{
${ }_{12}$ «Veit lo romi qui trait e met sablun El sac qu'ele li tent a geneillun [...] Vit sa muillier Bertran qui tent lo sac; Cil i met lo sablun que trait del brac». Ibidem, v. $9742-9743$ et v. $9757-9758$.

${ }^{13}$ «Quant de Sainte Sufie fist reis moster, Si defendie la gent de son enper, E de Costantinoble a toz lor quer, Que uns nen i mesest vaillant dener[...] En conprave de l'erbe, que li sonmer Manjavent quant s'estavent desoz l'onbrer. La nuit, quant gent durmie, a son bocler Aportave de l'aigue sobre el morter». Ibidem, V. 9682-85 et v. 9689-9692.

${ }^{14}$ Gesta abbatum sancti Germani Autissiodorensis, cap. XXVII, reconstruction de l'église du monastère sous l'abbé Jean: «Adveniente ergo tempore, cum propter incohationem novis operis necessarium foret vetus destruere, ne thesaurus pretiosissimus reliquiarum, qui ibidem in majore altari repositus fuerat ab antiquo, minus digne minusque reverenter inter laïcos operarios tractaretur, maxime cum luminaria, ut decet, ibidem non possent accendi, nec missarum consueta solemnia celebrari, decretum est ut eaedem reliquiae ad alium locum ejusdem ecclesiae magis celebrem transferentur». V. MORTET, P. DESCHAMPS, Recueil de textes, op. cit. (n. 7), p. 78. Voir N. DEFLOU-LECA, G. LOBRICHON, Les gestes des abbés de Saint-Germain d'Auxerre, Paris, 2011, p. oo.

${ }_{15}$ «Quidam homo, nomine Fulco, pictoris arte imbutus, venit in capitulum Sancti Albani ante Girardum abbatem et totum conventum, et ibi fecit talem convenientiam. Pinget totum monasterium illorum et quidquid ei perceperint, et vitreas fenestras faciet. Et ibi frater eorum devenit et insuper homo

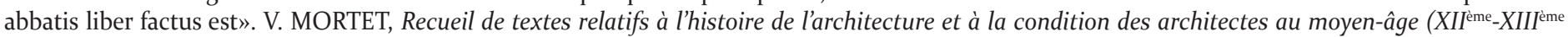
siècle), Paris, 1911, p. 264-265.

${ }^{16}$ V. MORTET, P. DESCHAMPS, Recueil de textes, op. cit. (n. 7), p. 289.
} 


\section{3-Le cas du monastère féminin}

La question se pose différemment dans le cas d'un monastère féminin, car la féminité impose une rupture plus radicale avec le siècle. Comme le veulent les premières règles pour monastère de femmes, les moniales doivent d'une part éviter le contact avec les hommes, d'autre part, le cas échéant, faire preuve alors de pudeur et de réserve. La Règle des vierges, édictée au VI ${ }^{\mathrm{e}}$ siècle par saint Césaire, évêque d'Arles, pour les moniales du monastère Saint-Jean, une règle très rigoureuse visant à préserver la pureté du monastère et sa bonne renommée, a, pour cette raison-même, prévu, au chapitre 36 , le cas des hommes devant œuvrer sur les édifices monastiques: «Ante omnia propter custodiendam famam vestram nullus virorum in secreta parte in monasterio et in oratoriis introerat, exceptis episcopis, provisore et presbytero, diacono, subdiacono et uno vel duobus lectoribus, quos et aetas et vita commendat, qui aliquotiens missas facere debeant. Cum vero aut tecta retractanda sunt, aut ostia vel fenestrae sunt componendae, aut aliquid hujusmodi reparandum, artifices tantum et servi ad operandum aliquid, si necessitas exegerit, cum provisore introerant; sed nec ipsi sine scientia, aut permissione matris. Ipse vero provisor, in interriorem partem monasterii, nisi pro his utilitatibus, quas superius comprehendimus, numquam introeat, et aut numquam aut difficile sine abbatissa aut alia honestissima teste: ut sanctae secretum suum, sicut decet et expedit, habeant $»^{17}$.

Le texte fait mention de la secreta parte du monastère, le lieu «séparé», le plus intime de la vie monastique. Malgré les impératifs de pudeur, la règle admet que des hommes, habilités par leur fonction dans l'Eglise et leur réputation morale (exceptis episcopis, provisore et presbytero, diacono, subdiacono et uno vel duobus lectoribus), puissent y pénétrer, pour les messes. A cette liste, Césaire ajoute celle des hommes de l'art et de leurs serviteurs (artifices tantum et servi) qui ont à intervenir sur les édifices formant le cœur de la vie des moniales. Le texte n'envisage pas les chantiers de construction ou de reconstruction, mais seulement les travaux de réparation. La liste ouverte qui en est donnée fournit des indications sur les interventions le plus cou- rantes: elles concernent des toits à reprendre, des portes et des fenêtres à installer (cum vero aut tecta retractanda sunt, aut ostia vel fenestrae sunt componendae) ou, précise l'article, d'autres travaux du même ordre (aliquid hujusmodi reparandum). Hommes de l'art et serviteurs peuvent alors entrer, accompagnés du proviseur. Il s'agit là d'une remarquable reconnaissance des nécessités pratiques liées à l'entretien des bâtiments et au confort des moniales. Le texte est très précis et propose une courte casuistique. Le critère d'admission au sein de l'espace monastique, c'est les connaissances techniques qui justifient le recours aux artisans et à leurs aides (sed nec ipsi sine scientia). Le proviseur lui-même ne peut accéder au cœur du monastère en dehors des travaux à réaliser, sauf à être accompagné de l'abbesse ou de sa représentante. On se demande dès lors qui, dans ces conditions, peut surveiller l'évolution du bâti et décider des réparations à opérer. Sans doute le proviseur, régulièrement accompagné.

\section{4-Les dangers du chantier}

Mais l'intimité monastique ou la pudeur féminine ne sont pas les seules causes des dispositions visant à séparer artisans et moines ou moniales. Le chantier est aussi un lieu dangereux et l'on peut imaginer que les religieux devaient s'en tenir éloignés. Si les relations de catastrophes, comme l'incendie de l'abbatiale de Tournus ${ }^{18}$ en 1007-1008 ou ceux de Vézelay et de Saint-Front de Périgueux ${ }^{19}$ en 1120, mettent parfois en scène la mort de moines et de fidèles, les récits de construction en revanche évoquent plutôt les accidents mortels des hommes de l'art, souvent laïcs, mais appartenant parfois aussi au monde monastique, comme Odolric ${ }^{20}$, moine-peintre prêté par Saint-Julien de Tours à l'abbaye de Saint-Benoît-sur-Loire. Toutefois, il faut rester prudent. On a parfois interprété des événements mortels tels que la chute d'une planche depuis le clocher de la priorale de Paray-leMonial sur un jeune moine au temps d'Hugues de Semur, comme la preuve d'un accident de chantier et de la présence de moines sur les lieux des travaux. Il est plus raisonnable d'y voir la simple mention d'un incident dû à la vétusté du

\footnotetext{
${ }^{17}$ CESAIRE D'ARLES, Règle des vierges, in ID., CEuvres monastiques, I, texte et traduction d'A. de Vogüé et J. Courreau, Paris, 1988 (Sources chrétiennes, 345), p. 170-273, cap. XXIII, chap. 36. La Règle de Donat, règle féminine composée au VIIe siècle d’après les règles de saint Césaire, de saint Benoît et de saint Colomban par saint Donat, évêque de Besançon, pour le monastère bisontin de Jussamoutiers, reprend, dans son chapitre 55 (Comment les procureurs du monastère et d'autres hommes doivent entrer dans le monastère), les recommandations du chapitre 36 de la Règle des vierges: «1. Avant tout, veillez à votre réputation, et n'autorisez un homme à pénétrer dans les parties retirées du monastère ou dans l'oratoire, 2. exception faite pour les évêques, le prêtre et le diacre et un ou deux lecteurs recommandables par leur âge et leur conduite, qui de temps en temps doivent célébrer la messe. 3. Quand la toiture, les portes et les fenêtres devront être refaites, ou pour toute autre réparation, les ouvriers seuls et leurs aides entreront avec le procureur, si c'est nécessaire ; 4. et cela seulement au su de la mère et avec son autorisation. 5. En dehors de ces cas de nécessité, le procureur lui-même n'entrera à l'intérieur que pour les travaux mentionnés ci-dessus, et jamais ou exceptionnellement, sans l'abbesse ou une autre sœur irréprochable comme témoin : 6. afin que les femmes consacrées à Dieu gardent leur vie cachée, comme il sied et il convient». V. Dupont, L. de Seilhac (ed.), Règle de Donat, in L. DE SAILHAC, M. BERNARD SAÏD, coll. M. BRAQUET et V. DUPONT, Règles monastiques au féminin, Bégrolles-en-Mauges, 1996, p. 99-176 (159).

${ }_{18}$ «Consumpta igitur, ut dictum est, prememorato in incendio nostrarum non minima parte rerum, in quo etiam Odo atque Morinus, duo videlicet pueri vicissim ses cohortantes ut a sanctuario non egrederentur, ne tante superstitis essent calamitati, perisse decentur». FALCON, Chronicon trenorciense, 4546, texte cité par P. JUENIN, Nouvelle histoire de l'abbaye royale et collégiale de Saint-Filibert et de la ville de Tournus, Dijon, 1733, preuves p. 26.

${ }^{19}$ «Anno D. MCXX XI kalend. augusti, monasterium S. Mariae Magdalenae de Vizeliaco combustum est cum mille centum viginti et septem hominibus et feminis; similiter incensum est monasterium Sancti Frontonis civitatis Petragoricae cum multis hominibus et feminis». La chronique de Saint-Maixent (751-1140), éd. et trad. J. Verdon, Paris, 1979, p. 192.

${ }^{20}$ Vita Gauzlini, abbatis Floriacensis monasterii, § 56: «Interea beati Petri apostoli aecclesia incendio, ut diximus, concremata, senioque annorum defessa, icta ventorum flabris, a fundamento corruit. Que in melius constructa, aliptico scemate est eleganter deflorata a quodam sanctimartyris Juliani Turonice sedis monacho, nomine Odolrico. Hic, dum una dierum operis accelerandi inservit, vocatur ab eodem dilecto Christi. Qui, dum a vimineo festinat descendere ambulatorio, rastrorum fidens scapulis, quibus ascensus descensusque sat continuabatur difficilis, uno eorum eliso, inter ipsius manus preceps humi dilabitur ; primoque podiis ipsius aecclesie mediestinus inliditur, ad extremum solo ac si moribundus deturbatur. Sed, paululum resumpto spiritu, a terra prosilit, signo se crucis munit, et, quis eum subridens deposuisset cum clamatione interrogat frequenti, quia non solum omni corpore incol[u]mis, verum quolibet dolore apparebat extraneus omni modis». Vie de Gauzlin, abbé de Fleury, texte éd., trad. et annoté p. R.-H. Bautier et G. Labory, Paris, 1969, p. oo.
} 
beffroi ou à son manque d'entretien, comme on le constate pour le clocher de l'abbatiale de Saint-Benoît-sur-Loire ${ }^{21}$. Il arrive aussi que ces récits, souvent miraculeux ${ }^{22}$, révèlent la présence d'individus, attirée par les cris des blessés ou des témoins. Parmi les miracles de saint Benoît rédigés par Raoul Tortaire, celui qui concerne le dénommé Otgerius ${ }^{23}$, tué par la chute d'une poutre, décrit l'arrivé sur les lieux d'une foule de moines et de laïcs («Accurrimus statim multitudo tam laicorum quam monachorum»), qui entrent dans le chantier sans aucune difficulté, comme si les seules barrières de protection étaient morales.

\section{TYPOLOGIE DES CHANTIERS}

L'installation d'un chantier de construction dans un espace monastique généralement encombré constitue l'autre aspect de la question. Le problème qui est alors posé concerne précisément la concurrence des travaux et des activités liturgiques au cœur du monastère: comment assurer la pérennité des messes et des offices, quand l'église majeure, qui en concentre l'essentiel, est en reconstruction? La durée de l'opération, habituellement longue, oblige l'abbé constructeur à mettre en place des stratégies spécifiques. Didier, abbé du Mont-Cassin (1058-1087), a mis habilement à profit la topographie religieuse du site pour organiser une utilisation tournante des édifices de culte ${ }^{24}$. Il fait d'abord reconstruire l'église Saint-Pierre, un vaste édifice axé nordsud et située près de l'infirmerie. Puis, après avoir ouvert l'église fraîchement achevée aux messes et aux offices, il fait édifier une abbatiale neuve sur le modèle de l'ancienne ${ }^{25}$.

Mais en règle générale, quand il s'agit de reconstruire une église, on adopte une des deux formules couramment employées pour associer sans encombre l'église vieille et l'église neuve. Dans le monde religieux, et dans les monas- tères en particulier, on rencontre trois types de chantier, définis par les relations entretenues - ou non - par l'église en construction avec l'église à remplacer: le chantier allotopique, le chantier hétérotopique et le chantier homotopique. L'adjectif allotopique caractérise un chantier établi loin de l'église vieille. Cet éloignement explique l'absence de contraintes ou d'influences formelles de l'un sur l'autre. C'est pourquoi il n'en sera pas question ici. L'adjectif hétérotopique s'applique à un chantier ouvert à côté de l'église à remplacer. D'éventuelles relations de nécessité peuvent expliquer des contraintes exercées par l'église vieille sur l'église en construction. L'adjectif homotopique qualifie un chantier installé sur le site même de l'édifice à reconstruire. La construction de l'église neuve, prévue plus grande que l'ancienne, débute à l'extérieur de l'église vieille, plus petite, qui reste debout pour que soit assurée la continuité des cérémonies religieuses. Puis, lorsque le sanctuaire neuf est disponible, le sanctuaire vieux est détruit. Enfin, la construction de la nef fait disparaître, progressivement ou d'un coup, la nef vieille. Cette démarche est susceptible de prendre les formes le plus diverses.

\section{1-Le chantier hétérotopique}

La construction de l'église neuve à côté de l'ancienne peut prendre deux formes. Le plus souvent, les deux édifices sont localisés côte à côte, en parallèle (chantier hétérotopique parallèle). Parfois - les exemples sont plus rares, mais bien attestés - l'assiette de l'église neuve pivote de 90 degrés (environ) et l'axe du nouvel édifice se retrouve à angle droit par rapport à celui de l'ancien (chantier hétérotopique pivoté). Le cas est illustré par la reconstruction de l'église Saint-Bonaventure de Lyon au XIVe siècle ${ }^{26}$. Les franciscains se sont implantés entre Saône et Rhône ${ }^{27}$ dans les années 1220. Ils

${ }^{21}$ Accident de Paray-le-Monial: «Talis siquidem modo occultatum fuit quod apud Paredum fecit pietatis exibitor. Turris in quam signa in medio dependentia consistur compage soluta puerum scolarem qui suberat pene extinxit. Tabula enim de culmine ipsius collapsa innocentem percussit, strauit, et ut uidebatur exanimauit. Dolorem impendunt qui consulere nesciunt, nunciantque patri puerum iam extinctum qui in miliciae spifitualis procinctu uix viuere inchoasset. Accessit pius uisitator et corpusculo confracto adhibens fomentum caelestis gratiae ad menbra emortua tenuem flatum reuocauit. Protinus decumbentem in statum erigens nomine conclamatum suo excitavit et pristinae restituit sanitati». Gilo, Vita sancti Hugonis abbatis, I, XXXII, in H.E.J. COWDREY, Two Studies in Cluniac History, I. Memorials of Abbot Hugh of Cluny (1049-1109), in Studi Gregoriani, 11, 1978, p. 77. Accident de Saint-Benoîtsur-Loire: «Tertius etiam, Archembodus vocitatus, dum, ad dependenda majora signa, recenter beatae memoriae [...] constructa industria, quoddam lignum aptaret, a superiori turris lapis immensi decidit ponderis, miserumque inter scapulas ictum pene exanimem reddidit. De ejus vita desperatis omnibus, ad domum propriam semivivus defertur ; cujus infortunio, beatus pater, comperto, accessit ad eum per semetipsum, et infirmi casibus, tanquam in se susceptis, compatiebatur [...] Sciensque scriptum esse: «Quanto magnus es, in omnibus humilia te et coram Deo invenies gratiam, nullum pretermittit diem, quin predictum visitet languentem. Victus subministrat alimoniam, et quasi operi presentem cotidiani laboris largiri precipit mercedem. Verumtamen expleto unius septimane spatio, pristine jam redditus medele, gratiarum actiones reddit pro voto, deincepsque aecclesiastico se letabundus mancipat servitio"». Vita Gauzlini, abbatis Floriacensis monasterii, § 54, in Vie de Gauzlin, abbé de Fleury, op. cit. (n. 20), p. oo.

${ }^{22}$ L'accidenté échappe à la mort ou en revient miraculeusement.

${ }^{23}$ «Ergo, dum quodam mane, trabibus tenui imbre madefactis, operarii desuper incederent, praeparantes quae praecipiebantur, unus eorum, Otgerius nomine, minus caute superambulans, dum affigere vestigia uni trabium fortiter nititur, pede lapso de illa celsitudine per compaginatos sibi fustes, per multiplicem lignorum struem, inter acervos lapidum pronus ad terram corruit. Erat enim pene totum illud telluris spatium tam lignorum quam saxorum congerie adopertum, parvus tantum locellus ad mensuram humanae proceritatis remanserat immunis ab eis. In quo admirantibus qui aderant, ac si de industria proiectus decidit juvenis ipse. Attrito tamen capite soli duritie, oculis pene erutis, lingua ab ore porrecta, perfusus faciem rivo cruoris, jacebat humi tanquam exanimis. Accurrimus statim multitudo tam laicorum quam monachorum qui, etsi casum non videramus, clamoren tamen et luctum artificum audieramus». Les miracles de saint Benoît, écrits par Adrevald, Aimoin, André, Raoul Tortaire et Hugues de Sainte-Marie, moines de Fleury, réunis et publiés par E. De Certain, Paris, 1858, p. 328.

${ }^{24}$ M. ANDALORO, L’abbaye du Mont-Cassin, sur les traces d'un édifice disparu, Chantiers médiévaux, Paris-La Pierre qui vire, 1996, p. 53-70.

25 «Anno ordinationis itaque sue nono, divine autem incarnationis millesimo sexagesimo sexto mense Martio indictione quarta constructa prius juxta infirmantium domum non satis magna beati Petri basilica, in qua videlicet fratres ad divina interim officia convenirent, supradictam beati Benedicti ecclesiam tam parvitate quam deformitate thesauro tanto tanteque fratrum congregationi prorsus incongruam evertere a fundamentis aggressus est». LEONE MARSICANO, Cronaca di Montecassino (III, 26-33), a cura di F. Aceto e V. Lucherini, Milano, 2001, p. 44. L'abbatiale a été démolie en 1066 et la nouvelle consacrée le $1^{\text {er }}$ octobre 1071.

${ }^{26}$ N. REVEYRON, De la première à la seconde église des cordeliers, in L'église Saint-Bonaventure au cœur de la vie lyonnaise, dir. M. Quesnel, Lyon, 2016, p. 21-35.

${ }^{27}$ H. CHOPIN, Une église dans une ville, les origines de l'église Saint-Bonaventure, Ibidem, p. 10-15. 


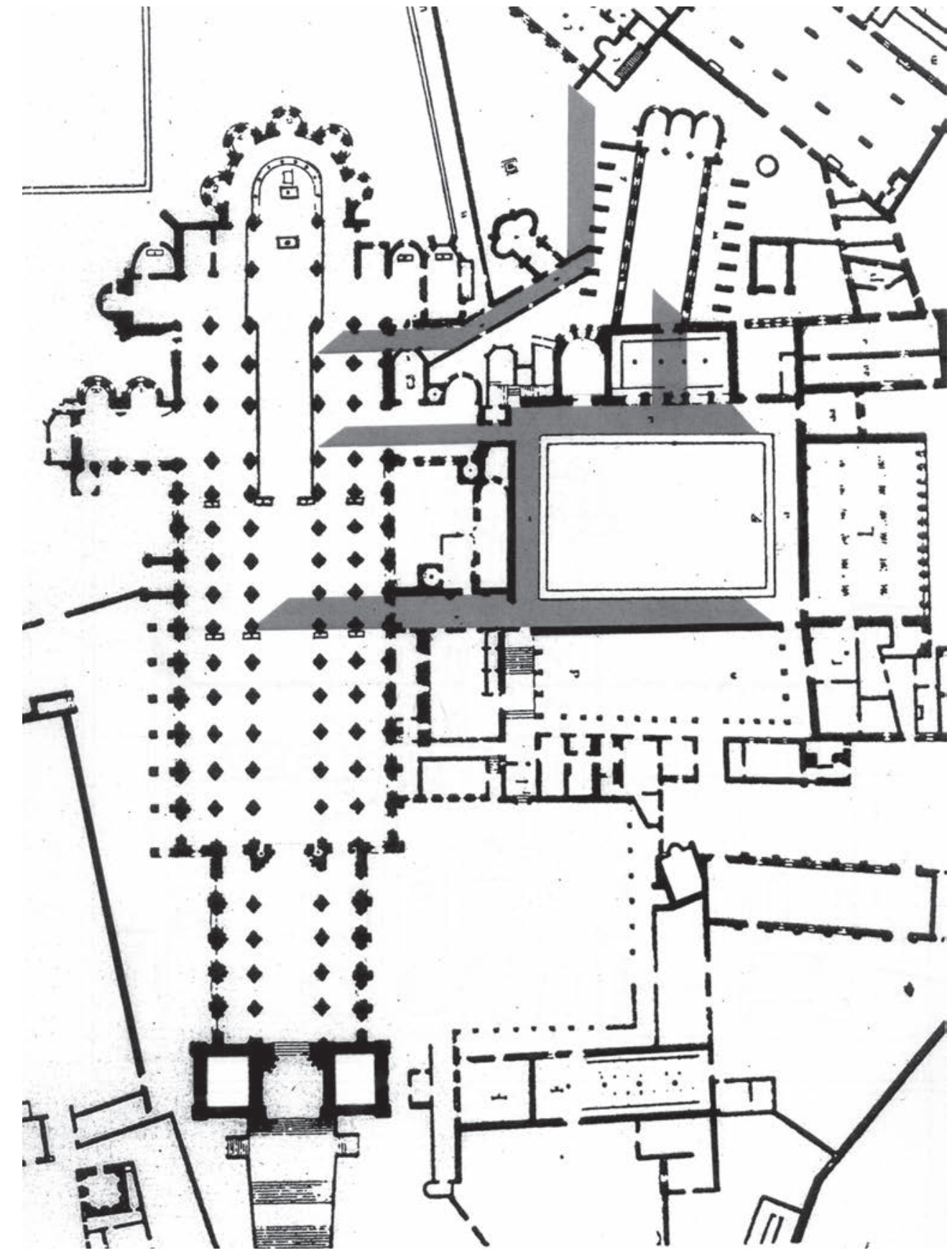

Fig. 1. Cluny, d'après le plan, de 1700, avec indication des circulations majeures vers l'abbatiale. pas. Ils ont alors aménagé une grande place au nord, devant la façade, et sur le flanc ouest de l'église.

De fait, pour des institutions soucieuses des populations, comme les mendiants ou les hospitaliers, l'adaptation à la ville des bâtiments, $\mathrm{y}$ compris l'église, est un impératif majeur, qui peut avoir des conséquences contraires aux habitudes, comme celle de l'orientation. Le choix du lieu de la nouvelle implantation révèle quelque chose de l'étroite articulation entre l'implantation de l'église nouvelle, l'organisation du réseau viaire et les questions d'architecture monumentale. Dans le domaine des hospitaliers, le phénomène est illustré par le cas de l'hôpital de Santa Maria dei Battuti, à San Vito al Tagliamento, dans le Frioul ${ }^{28}$. Etablie le long de la voie qui dessert la cathédrale, de l'autre côté, la première église du site hospitalier, qui remonte à 1360, est normalement orientée et dotée, sur le flanc sud, d'un long portique ouvrant directement sur la rue. $\mathrm{Au} \mathrm{XV}^{\mathrm{e}}$ siècle est construite, un peu plus à l'ouest, une autre église, plus vaste, axée nord-sud, dont la façade donne sur rue.

La construction de la troisième abbatiale de Cluny par Hugues de Semur à partir de 1088 est l'exemple le plus célèbre de chantier hétérotopique en parallèle (fig. 1). Ici encore, les enjeux sont importants, d'autant plus importants que le dense tissu de constructions et de réseaux de circulation dans l'abbaye apportent des contraintes lourdes. Au moment où débutent les travaux, le cœur de l'abbaye est constitué de quatre éléments en étroite interrelation: ont construit leur première église sur le côté sud du tènement qui leur a été concédé, perpendiculairement au Rhône, qui coule à l'est, et l'abside tournée vers le fleuve. Dans le dernier tiers du XIV ${ }^{\mathrm{e}}$ siècle, ils reconstruisent un édifice très long sur le côté ouest de leur terrain, et sur un axe nord-sud. Les deux églises n'ont aucun point de contact. Toutes deux répondent cependant à la même exigence d'être positionnées non loin d'une entrée de la ville et près des axes importants de communication. Mais le choix du nouveau site répond visiblement à une évolution du contexte urbain. Les frères mineurs ont en effet souhaité insérer plus étroitement le monastère dans la ville et ouvrir à la population des espaces libres autour de l'église, ce que l'ancien site ne permettait la vieille abbatiale de Cluny II, la salle capitulaire, l'église mariale et l'infirmerie. Si chacun de ces édifices possède ses fonctions propres, ils composent par groupe ou tous ensemble des lieux en réseau dédiés à des liturgies spécifiques $^{29}$. L'abbatiale, le chapitre et l'église mariale abritent successivement le matin les prières quotidiennes pour les morts. Avec l'infirmerie, le chapitre et l'église mariale participent pleinement à l'accompagnement des mourants: le malade et le convent empruntent à plusieurs reprises l'église mariale pour se rendre du chapitre à la chambre du mourant et réciproquement. Mais l'abbatiale intervient aussi au moment de la dernière communion. Par ailleurs, d'autres contraintes topographiques ont pesé sur le choix, notam-

\footnotetext{
${ }^{28}$ F. METZ, L'assistenza sanitaria in San Vito al Tagliamento, L'ospedale di Santa Maria dei Battuti, dalle origine al XX secolo, Pordenone, 1993.

${ }^{29}$ En dernier lieu, voir F.S. PAXTON, Death by Customaries at Eleventh-Century Cluny, en S. Boynton et I. Cochelin (éd.), From Dead of Night to End of Day, du cœur de la nuit à la fin du jour, les coutumes clunisiennes au Moyen Age, Turnhout, 2005, p. 297-318; N. REVEYRON, Hugues de Semur et l'architecture clunisienne, Influences de la liturgie et des coutumes monastiques sur les programmes architecturaux dans l'ecclesia cluniacensis, in Monuments et mémoires de la Fondation E. Piot, 2012, p. 91-147.
} 
ment la présence d'un cimetière monastique ancien au nord de Cluny $\mathrm{II}^{30}$. Dans ces conditions, il était difficile d'établir le chantier de construction autour de la vieille abbatiale. En revanche, il était nécessaire de prévoir l'intégration de la nouvelle abbatiale aux réseaux de circulation. Cluny III a été positionné de telle sorte que le grand transept, prévu pour mener les moines du futur cloître au chœur monastique, fût implanté dans le prolongement de la galerie orientale du vieux cloître, que la masse de la vieille abbatiale séparait encore du chantier de la nouvelle. Par ailleurs, le petit transept a été doté d'une porte qui devait permettre au prêtre portant le viatique au moine mourant d'aller directement de Cluny III à l'infirmerie, sans passer par l'église mariale ${ }^{31}$.

\section{2-Le chantier homotopique}

Comme le chantier hétérotopique, le chantier homotopique présente deux dispositions types: soit le chantier de la nouvelle église entoure l'église vieille, suivant le même axe, soit il se développe selon un axe perpendiculaire (chantier homotopique pivoté) ${ }^{32}$, tout en englobant l'ancien édifice dans la masse du nouveau. Le second type concerne-t-il tout particulièrement franciscains ou les dominicains, comme on l'a vu précédemment pour les chantiers hétérotopiques et comme on le verra plus loin avec Notre-Dame-de-Confort (Lyon) ou San Francesco de Pistoia? En l'absence d'études élargies, il est difficile de l'affirmer. Mais il est vrai que l'installation des ordres mendiants dans un tissu urbain déjà dense, les formes souvent étriquées des terrains qui leur sont donnés et l'existence parfois d'une église ancienne sur ce terrain peuvent expliquer le phénomène.

Implantés à Lyon en 1218 au bas de la montée du Gourguillon, au sud de la cathédrale, les dominicains s'installent dans la presqu'île en 1236, à la limite sud de la ville, sur un terrain donné par l'abbaye d'Ainay ${ }^{33}$. Ils héritent d'une petite église orientée nord-sud dont ils font le vestibule de leur nouvelle église, Notre-Dame-de-Confort, commencée en 1244 et consacrée par Innocent IV en 1251. L'intérêt présenté par cette sorte d'avant-nef de deux vaisseaux et trois travées est de créer l'entrée de la nouvelle église à la place du mur nord de la travée nord de l'église vieille, face au débouché de la rue Mercière, l'artère principale de la presqu'île. C'est la démarche inverse qui a présidé à l'implantation de l'église franciscaine de Pistoia, en Toscane. La vaste église San Francesco a pris la succession de la petite église Santa Maria Maddalena al Prato, cédée 1248 aux franciscains par les chanoines de la cathédrale ${ }^{34}$. Santa Maria était normalement

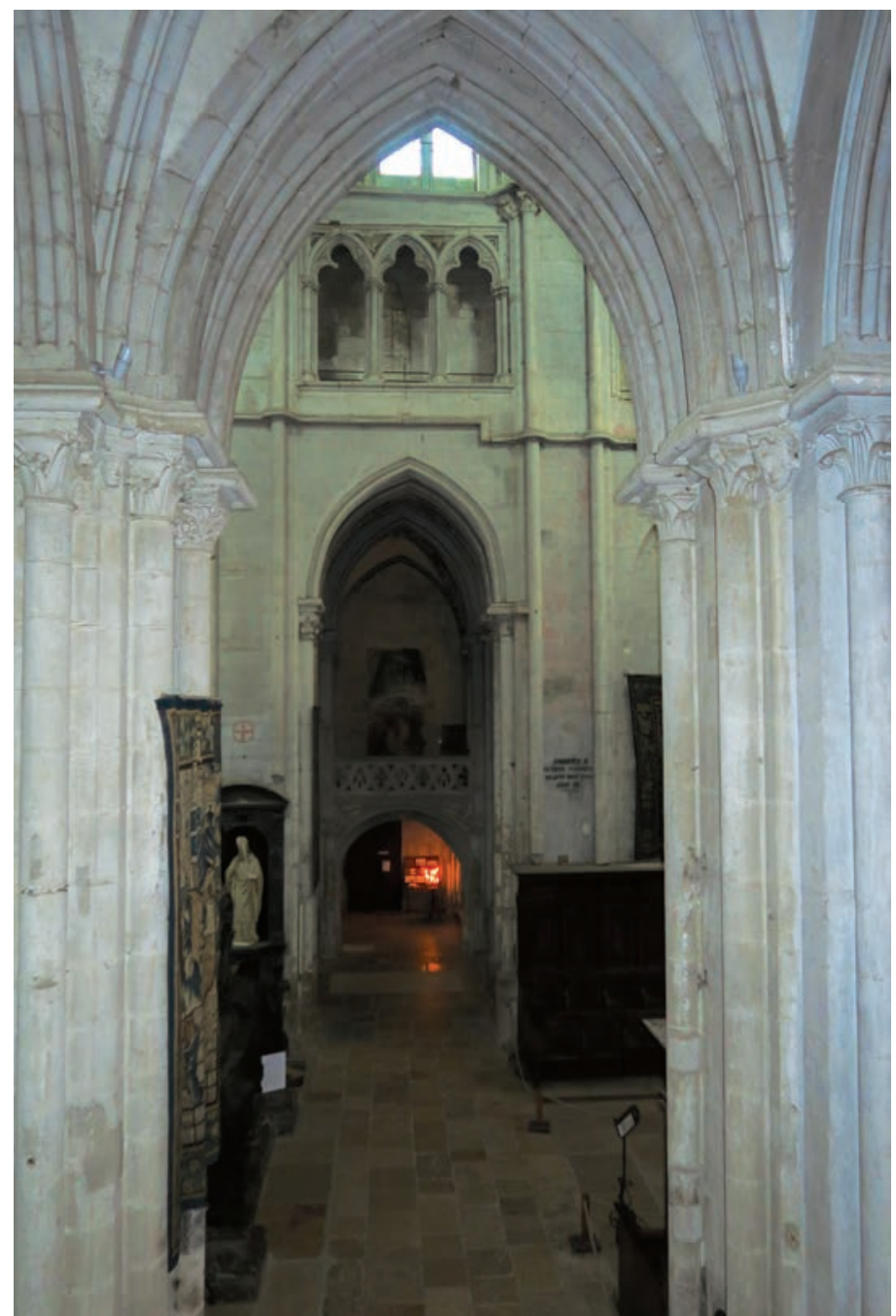

Fig. 2. Saint-Antoine-en-Viennois, abbatiale: vue du bras nord du transept, depuis le bras sud. Cliché de l'auteur.

orientée, San Francesco, commencée en 1298, est tournée vers le sud, comme Saint-Bonaventure de Lyon. La construction s'est déroulée de façon inhabituelle de la façade vers l'abside, mais on ignore si l'érection de la façade a fait disparaître immédiatement Santa Maria ou si celle-ci a été conservée aussi longtemps que les frères mineurs n'avaient pas de chœur à leur disposition.

Le phénomène, à défaut de son nom, a été curieusement théorisé en 1534 par Aymar Falco dans son histoire de l'abbaye de Saint-Antoine-en-Viennois ${ }^{35}$. Personnage important de l'ordre, historien scrupuleux, malgré des par-

\footnotetext{
$3^{\circ}$ A. BAUD, La place des morts dans l'abbaye de Cluny. État de question, in Archéologie Médiévale, 29, 2000, p. 99-114. Ce site funéraire avait gardé une réelle importance, puisqu'y a été construit au XII 'e siècle sans doute un Saint-Sépulcre. K.J. CONANT, Cluny, les églises et la maison du chefd'ordre, Mâcon, 1968, p. 39. ${ }^{31}$ A propos du transport par le prêtre du Saint Sacrement depuis l'abbatiale jusqu'à l'infirmerie, F.S. PAXTON, op. cit. (n. 29), a montré que le coutumier du temps d'Odilon, le Liber tramitis aevi Odilonis, présentait deux ordo différents. Selon le premier ordo, le prêtre devait emprunter le chemin habituel, c'est-à-dire passer par le chapitre et l'église mariale. Le second ordo exprime un souhait contraire, c'est-à-dire aller directement de l'abbatiale à l'infirmerie. ${ }^{32}$ En réalité, l'angle que la nouvelle église fait avec l'axe de l'ancienne peut être égal ou inférieur à 90 degrés, mais dans tous les cas, il répond à un changement très marqué d'orientation.

${ }_{33}$ Actuelle place des Jacobins. Sur l'histoire des dominicains de Lyon, voir M. CORMIER, L'ancien couvent des dominicains de Lyon, I. Description, plan, vues diverses, Lyon, 1898-1900; J.-D. LEVESQUE, Les frères prêcheurs de Lyon, Notre-Dame de Confort, 1218-1789, Lyon, 1978. Le plan commenté du couvent et de son église a été établi par le père Ramette en 1719 et publié par M. Cormier, op. cit., p. 11-40; première église: notice 86) et une description précise en a été retrouvée par Haude Morvan dans les Archivio Generale dell'Ordine dei Predicatori, XIV liber M, fol. 429-503: «Excerpta ex tabulario conventus Lugdunensis ordinis Praedicatorum monumenta quae annalibus conscribendis inservire possunt». Voir H. MORVAN, Les sépultures dans la propagande des frères prêcheurs et mineurs: quatre sépultures de cardinaux à Lyon au XIII e siècle, in Mélanges de l'École française de Rome. Moyen Âge [En ligne], 126, 1, 2014.

${ }^{34}$ Exemple cité par C. BRUZELIUS, The Architecture of the Mandicant Orders in the Middle Ages: an Overview of Recent Littérature, in Perspective, 2, 2012, p. 365-386. Voir S. Francesco: la chiesa et il convento di Pistoia, ed. L. Gai, Ospedaletto, 1993.

35 A. FALCO, Antonianae Historiae Compendium, Lyon, chez T. Payen, 1534.
} 
tis pris historiques, Aymar Falco est un auteur fiable, qui présente toujours ses sources, écrites ou orales. Selon lui, Jocelyn, seigneur de La Motte-aux-bois (Saint-Antoine-enViennois), avait rapporté de Constantinople en 1070 le corps de saint Antoine, qu'il voulait déposer dans un édifice digne du saint ermite ${ }^{36}$. Avec la petite église du village, axé nordsud, il résolut d'en construire une plus grande, en gardant la première comme transept de la seconde, tout en élevant un bâtiment provisoire en bois à l'emplacement du chœur actuel de Saint-Antoine, pour accueillir les reliques le temps du chantier. L'abbatiale du XIII ${ }^{\mathrm{e}}-\mathrm{XV}^{\mathrm{e}}$ siècle ne révèle aucune trace archéologique de cette histoire monumentale, si l'on excepte les formes inattendues du transept (fig. 2), dédié à Marie comme l'église primitive, et du chœur. Mais le récit rapporté comme une tradition orale a toutes les apparences du réalisme, sinon de la réalité, et justifie point par point les formes de l'église actuelle inadaptées à la contemplation des reliques.

\section{3-Les exemple de Nantua et de Paray-le-Monial}

Quand il concerne deux édifices - l'ancien et le nouveau - semblablement orientés, le chantier homotopique peut se présenter selon deux types différents. L'édifice à construire est établi soit sur le même axe que l'ancien (chantier homotopique d'axe), comme à Paray-le-Monial, et il débordera celui-ci sur tout son périmètre. Soit sur un axe décalé vers le sud ou le nord par rapport à celui de l'ancien (chantier homotopique décalé), comme à Nantua, et l'un de ses murs goutterots viendra se superposer à un mur goutterot de l'ancien.

\subsection{1-La priorale Saint-Pierre de Nantua}

La reconstruction de Saint-Pierre de Nantua illustre le cas du chantier homotopique décalé. L'abbaye de Nantua, dans le diocèse de Lyon, a été établie au haut Moyen Age dans la région froide et montagneuse du Bugey (Nantua I). Le premier document conservé est un diplôme de Pépin le Bref, daté du 10 août 757 . Monastère royal ${ }^{37}$, il est confié en 852 par Lothaire Ier aux évêques de Lyon ${ }^{38}$, puis est affilié à Cluny peu après l'an Mil. C'est à cette occasion, sans doute, que l'église est reconstruite (Nantua II), selon une formule très simple: une nef unique, terminée par une abside, à l'est (fig. 3). Hugues de Semur, qui aurait offert des reliques de saint Maxime, évêque de Riez, et un reliquaire couvert d'argent, en est le dernier abbé39: c'est sous son abbatiat sans doute qu'a été construite l'église actuelle (Nantua III). En 110o, le pape Pascal II réduit Saint-Pierre de Nantua au rang de prieuré, comme la majorité des monastères affiliés à l'abbaye bourguignonne. Mais l'église subit d'importantes modifications à la charnière du XII et du XIII e siècle.

L'église actuelle est un édifice de grande ampleur, composé d'une nef à trois vaisseaux de six travées, d'un transept

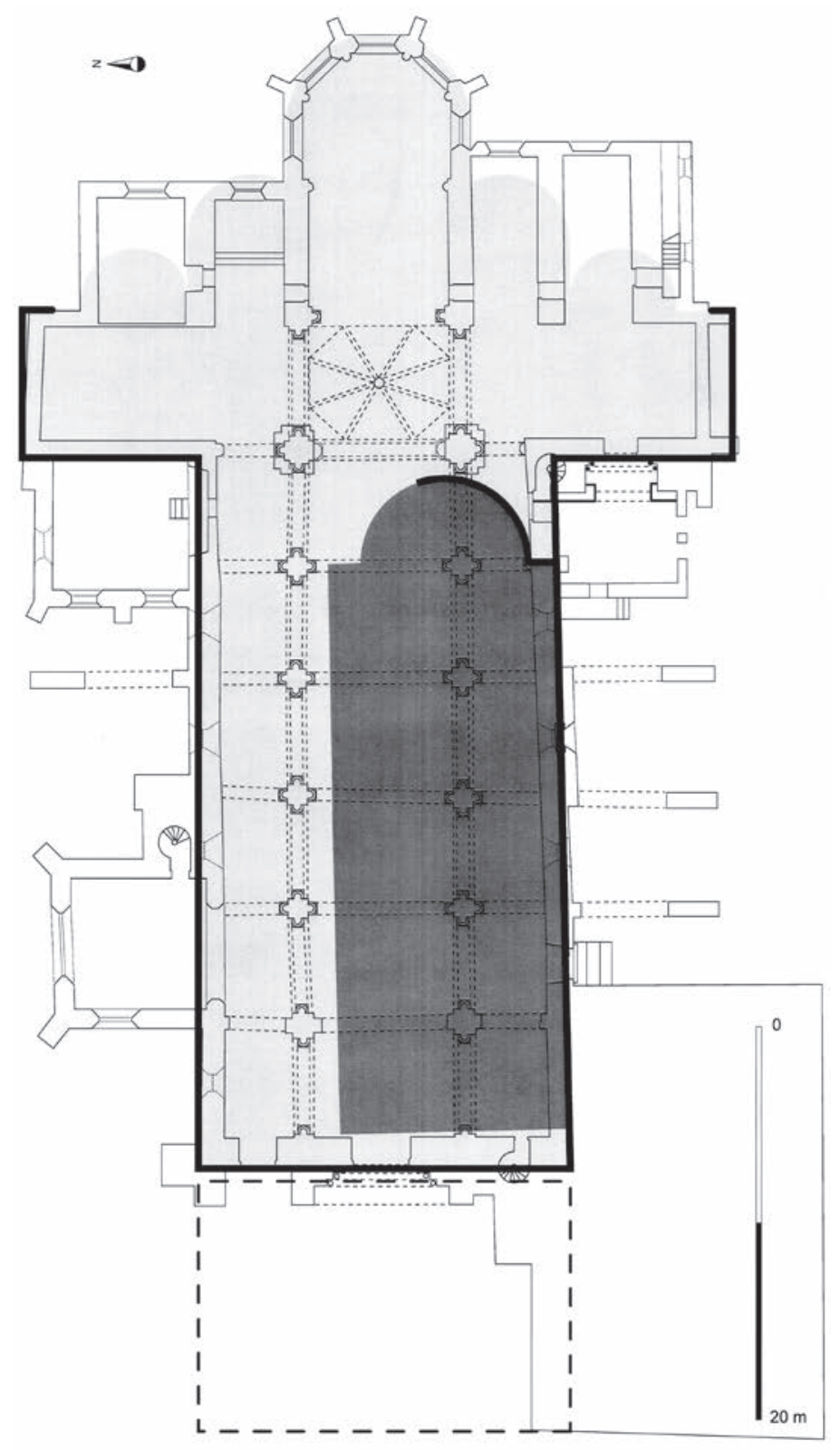

Fig. 3. Saint-Pierre de Nantua: superposition des plans de Nantua II et de Nantua III. DAO Y. Motmessin, laboratoire ARAR.

saillant greffé de quatre chapelles orientales et d'un sanctuaire profond (chœur et abside). La nef était à l'origine couverte d'une charpente. Les grands travaux du début du XIII ${ }^{e}$ siècle ont porté sur les couvertures (voûtes d'ogives sur le vaisseau central, voûtes d'arêtes sur les bas-côtés, voûte octopartite à la croisée), sur les accès majeurs (portail occidental et portail de la Vierge, faisant communiquer la galerie orientale du cloître avec le bras sud du transept) et sur le clocher (Nantua IIIb). La succession de Nantua II et de Nantua III s'est faite par le mur goutterot sud (fig. 3): celui de l'église neuve a repris le tracé (peut-être même les maçonneries) de celui de l'église vieille. Cette disposition

\footnotetext{
${ }^{36}$ A. FALCO, op. cit. (n. 35), secunda pars, cap. XXV, f. XLIIIr-v.

${ }^{37}$ J. DUBOIS, L'implantation monastique dans le Bugey au Moyen Age, in Journal des Savants, 1, 1971, p. 15-31 (18); M. RUBELLIN, Monastères et évêques dans le diocèse de Lyon aux IXe et $X^{e}$ siècles, in L'Eglise du IXe au XIe siècle, CAHMER, 3, 1991, p. 77-93 (80).

${ }^{38}$ L'histoire haut-médiévale de Nantua est marquée par un événement emblématique, mais sans conséquence pour ce qui nous intéresse: en 877 , le corps de Charles le Chauve, mort pendant son retour de Rome, est abrité dans l'abbatiale, le temps de préparer son transport à Saint-Denis; l'événement a marqué la mémoire de l'institution et ouvert des polémiques sur la géographie exacte de l'événement. J. NELSON, La mort de Charles-le-chauve, in Médiévales, 15-31, 1996, p. 55-66.
}

39 O. HUREL, D. RICHE, Cluny. De l'abbaye à l'ordre clunisien, $X^{e}-X V I I I^{e}$ siècle, Paris, 2010, p. 33. 


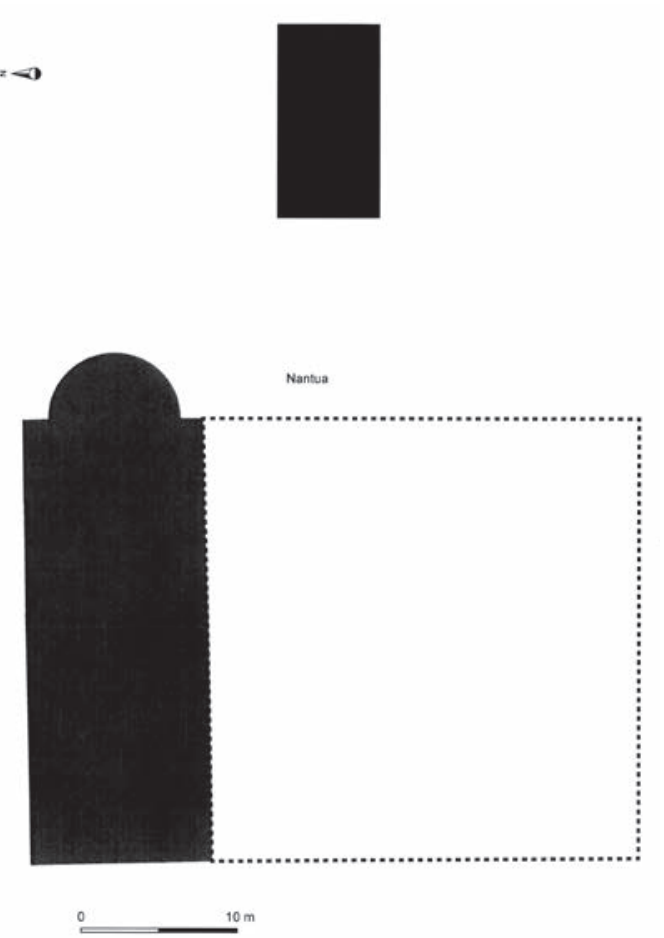

Fig. 4. Saint-Pierre de Nantua: restitution des édifices connues de Nantua II. DAO Y. Motmessin, laboratoire ARAR.

permettait d'assurer sans difficulté le passage d'un édifice à l'autre, sans toucher aux vieux bâtiments claustraux, situés au sud de ce même mur goutterot. Ces derniers, dont il ne subsiste rien, entouraient un cloître qui avait pour extension maximale la longueur de Nantua II, qui est aussi celle de la nef de Nantua III (fig. 4). Ils se trouvaient ainsi bien adaptés à la nouvelle priorale, y compris dans les portes menant de la galerie nord au bas-côté sud.

Ces bâtiments ont subi au moins des réaménagements, sinon une reconstruction. Des témoins significatifs de ces travaux subsistent dans l'angle du transept et du collatéral sud. Le portail de la Vierge, importante construction plaquée contre le mur ouest du bras sud du transept, répond à une importante fonction de circulation. Depuis la galerie orientale du cloître, elle donne accès soit au chœur ou à l'autel, soit à l'escalier qui, longeant la dernière chapelle sud du transept, mène directement à l'infirmerie, soit à l'église mariale par un passage ménagé dans le bras sud (fig. 5). L'iconographie mariale du tympan (adoration des Mages et songe de Joseph, en écho au portail occidental) est associée au thème de la porte et à la proximité de l'église mariale. Mais sur le massif très large du portail a été établie, dans la même phase du chantier, une discrète galerie de circulation qui conduit directement à l'étage de la galerie orientale, c'està-dire le dortoir. On y accède par un escalier en colimaçon

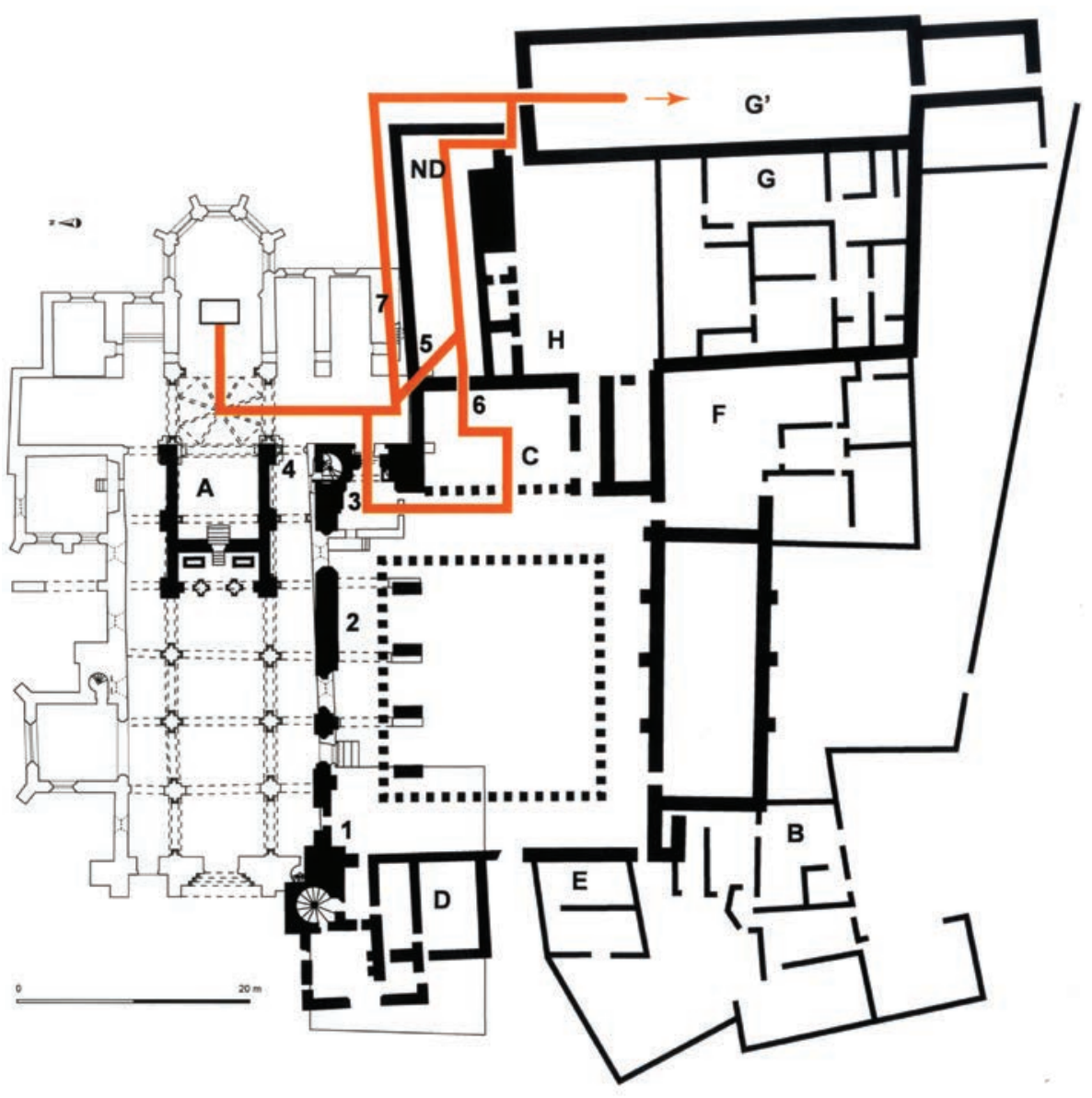

1 - Porte occidentale de la nef donnant dans le cloitre. 2 - Chapelle des Demoiselles et porte hypothétique du bas-chœur. 6 - Porte entre le chapitre et l'église mariale. 7 - Escalier du chevet, passage «du second ordo».

A - Chcour monastique. B - Maison du prieur commendataire nef. C - Chapitre. D - Maison de l'aumônier E - Maison du doyen. F-Maison du prieur claustral. G - Maison de linfirmier. $G^{\prime}$ - Infirmerie médiévale?

Fig. 5. Saint-Pierre de Nantua: restitution des circulations liturgiques dans l'abbatiale de de Nantua III. DAO Y. Motmessin, laboratoire ARAR

logé dans l'angle du transept et du bas-côté. Mais cet escalier se poursuit en hauteur, pour donner passage à l'intérieur du clocher (refait au XIX' ${ }^{\mathrm{e}}$ siècle).

\subsection{2-La priorale Saint Sauveur, Sainte Marie et Saint Jean-Baptiste de Paray-le-Monial}

La reconstruction de la priorale de Paray-le-Monial est un très bel exemple de chantier homotopique d'axe. Le monastère de Paray ${ }^{40} \mathrm{a}$ été fondé en 973 par Lambert, comte de Chalon, pour abriter une église dynastique et funéraire (Paray I) dont on ignore la forme et l'architecture ${ }^{41}$. Le fils du fondateur, Hugues de Chalon ${ }^{42}$, évêque d'Auxerre, en fait don à Cluny en 999. L'église est reconstruite au début du XI ${ }^{e}$ siècle (Paray II) et consacrée en 1004, selon Courtépée ${ }^{43}$. Son plan est ambitieux: une nef unique, à laquelle on ajoute une avant-nef vers 1080, un transept saillant à deux absidioles et un chœur profond terminé par trois absides (fig.

${ }_{40}^{\circ}$ M. CANAT DE CHIZY, Précis historique des origines du prieuré de Notre-Dame de Paray-le-Monial, Chalon-sur-Saône, 1876; F. NEISKE, Les débuts du prieuré clunisien de Paray-le-Monial, in Basilique de Paray-le-Monial: l'histoire, l'art, la vie, (Paray-le-Monial, 28-3o Mai 1992), Premier colloque international de Paray-le-Monial, mai 1992, Paray-le-Monial, 1992, p. 134-144.

${ }^{41}$ J. RICHARD, Les comtes de Chalon et leur sanctuaire dynastique, in Basilique de Paray-le-Monial, op. cit. (n. 40), p. 98-105.

${ }^{42}$ Hugues de Chalon est le grand oncle maternel d'Hugues de Semur, sixième abbé de Cluny et constructeur de Cluny III, ainsi que son initiateur à la vie spirituelle et monastique. M. CHAUNEY, Deux évêques bourguignons de l'an Mil: Brunon de Langres et Hugues $1^{e r}$ d'Auxerre, in Cahiers de Civilisation Médiévale, 21, 84, 1978, p. 385-393.

43 La date de l'historien de la Bourgogne converge avec les datations évoquées par l'archéologie. 


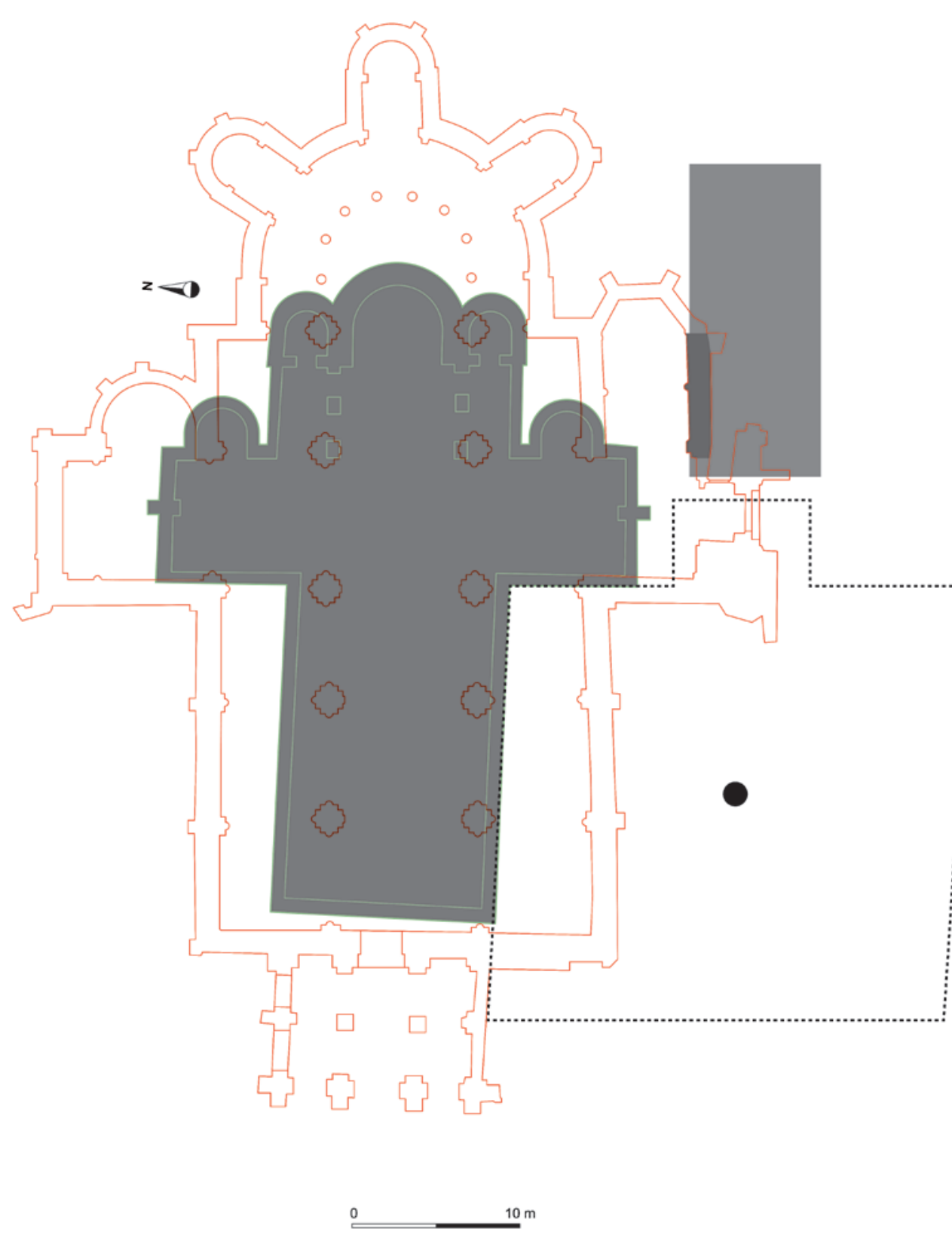

Fig. 6. Paray-le-Monial, superposition des plans de Paray II et de Paray III. DAO Y. Motmessin, laboratoire ARAR.

6) ${ }^{44}$. L'église est reconstruite au début du XIIe siècle sur le modèle de Cluny III et les travaux ont duré jusqu'au début du siècle suivant ${ }^{45}$, sans changement de parti, ni de style ${ }^{46}$.

L'organisation du chantier de Paray III est très révélatrice: la nouvelle église allait entourer exactement l'ancienne, les deux axes, déviés de quelques degrés l'un de l'autre, se croisant dans un lieu pivot très emblématique, l'emplacement de l'autel (fig. 6). Parallèlement, les deux transepts se recou- vraient exactement, le nouveau étant simplement plus long que l'ancien. On a d'abord construit le sanctuaire, développé à l'est du chevet de l'église vieille, avant d'en articuler les absidioles avec le transept vieux: pendant plusieurs décennies, faute de moyens, la priorale de Paray-le-Monial est restée cette église hybride, composée d'un chevet neuf de style antiquisant et du vieux transept et de la vieille nef. Les travaux se sont poursuivis par le nord: le mur périmétral de l'église s'est développé depuis le bras nord du transept jusquà la façade, l'avant-nef ayant été finalement conservée. La partie symétrique sud n'a été construite qu'en dernier: de fait, elle empiétait sur la galerie nord du cloître.

On peut tirer deux enseignements de cette disposition: d'une part, l'impact du chantier sur l'organisation des bâtiments claustraux du XIe siècle, d'autre part, le rôle du transept dans l'organisation du chantier. Certes, le cloître de Paray II ne nous est pas connu. On peut toutefois en estimer l'emprise au sol: plaquée contre la nef, la galerie nord devait en reproduire la longueur; quant aux autres galeries, elles devaient être construites en proportion. Parallèlement, la galerie orientale devait comporter la salle capitulaire. Selon la formule clunisienne, on l'a $\mathrm{vu}$, le chapitre s'ouvrait directement sur l'ecclesia beatae Mariae. Or, des textes du XVIII ${ }^{\mathrm{e}}$ siècle indiquent très clairement que cette église mariale se trouvait à l'extrémité sud-est du transept de Paray III, contre la chapelle du bras sud (actuelle chapelle de Damas-Digoine - fig. 6) (77 $^{4}$ cette disposition permet de localiser approximativement la salle capitulaire de Paray II, sous le bras sud de l'actuel transept.

La dernière phase des travaux de construction de Paray III, qui a concerné le bras sud du transept et le bas-côté sud de l'église neuve, a donc fait disparaître la galerie nord du cloître du XIe siècle, qui s'allongeait contre la nef de Paray

${ }_{44}$ Paray II est connu grâce aux fouilles menées par G. Rollier à la fin du siècle dernier. G. ROLLIER, La priorale du XIe siècle, in J.-N. BARNOUD, N. REVEYRON, G. ROLLIER, Paray-Le-Monial, Paris, 2004, p. 81-113 ; ID., Paray-le-Monial, Hugues de Semur: Lumières clunisiennes, Ibidem, p. 102-127; ID., Le projet dans le chantier roman de Paray-le-Monial, Le concept d'architecture clunisienne, in Archéopages, Grands travaux, 33, 2011, p. 66-73.

${ }_{45}$ N. REVEYRON, La priorale du XII e siècle: le chantier, in J.-N. BARNOUD, N. REVEYRON, G. ROLLIER, op. cit. (n. 44), p. 117-148. L'étude des élévations menée par G. Rollier à partir des échafaudages de restauration a confirmé les travaux précédents.

${ }^{46}$ Sur l'esthétique de Paray III: CH. OURSEL, Paray-le-Monial et Cluny, in Art Studies: Medieval, Renaissance and Modern, 4, 1926, p. 81-100; ID., L'art roman de Bourgogne, Dijon, 1926; ID., A.-M. OURSEL, Les églises romanes de l'Autunois et du Brionnais, Cluny et sa région, Mâcon, 1956; R. OURSEL, Bourgogne romane, La-Pierre-Qui-Vire, 1974; C.E. ARMI, Massons and Sculptors in Romanesque Burgundy: The New Aesthetic of Cluny III, University Park, 1983; M. KERR, The Former Cluniac Priory Church at Paray-le-Monial: a Study of its Eleventh and Twelth-century Architecture and Sculpture, Diss. Phil. Yale University, 1994.

${ }^{47}$ N. REVEYRON, Marcigny, Paray-le-Monial et la question de la chapelle mariale dans l'organisation spatiale des prieurés clunisiens au XI ${ }^{-}$-XII ${ }^{e}$ siècle, in Viator, 41, 2010, p. 63-94. 

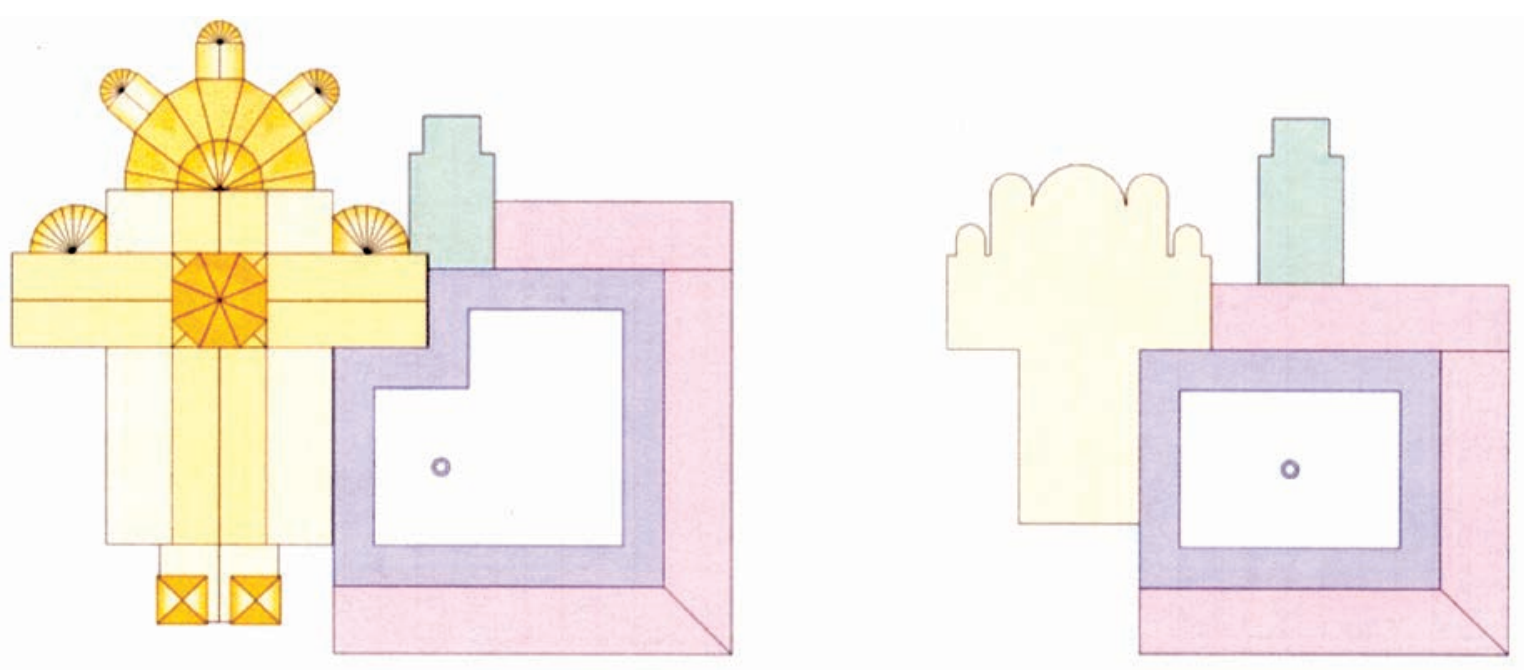

Fig. 7. Paray-le-Monial: le monastère de Paray II (à droite) et le monastère de Paray III (à gauche). 3D Baptiste Reveyron.

II, et tout ou partie de la galerie orientale, avec la salle capitulaire. Les difficultés posées par ces destructions, notamment celle du chapitre, touchent le quotidien du monastère et il faut se demander dans quelles nouvelles salles étaient transférées les activités indispensables. La localisation du nouveau chapitre (celui de Paray III) ne pose pas de problème. Comme le bras sud du transept actuel se termine par un portail donnant sur la galerie orientale du cloître, le chapitre neuf devait nécessairement être repoussé à l'est à la galerie et contre l'église mariale, pour les nécessités de communication $^{48}$.

Cette disposition met en lumière le rôle du transept neuf dans l'organisation du chantier (fig. 7). Tout d'abord, le nouveau transept est allongé outre mesure, au point que l'architecte lui a accolé, au nord et au sud, une travée supplémentaire, mal mariée avec le reste de l'élévation. Cet augmentum a pour finalité première de connecter physiquement le bras sud et l'église mariale, grâce à un passage dont la porte de l'actuelle sacristie est l'ultime avatar. Ensuite, le nouveau transept établit une liaison nord-sud qui permet d'intégrer le nouveau chapitre à l'ensemble du bâti réalisé. Enfin, le mur est du transept a constitué une sorte de barrière de chantier: d'un côté (à l'est), l'absidiole, l'église mariale et le nouveau chapitre, de l'autre, la vieille galerie orientale appelée à disparaître. Sans aucun doute, cette dernière n'a été démolie qu'après l'achèvement de la nouvelle salle capitulaire.

\section{CONCLUSION}

Les exemples de Cluny, Nantua et Paray-le-Monial montrent quel degré de raffinement ont atteint les stratégies de chantier, à peine perceptibles dans les textes. Le terme de stratégie n'est pas immérité: les constructeurs ont, d'une part, gardé le souci de reporter sur les générations suivantes les opérations le plus délicates qui aurait alourdi outrageusement l'engagement des premiers travaux, d'autre part, prévu, dans la distribution des ouvrages, l'agencement le meilleur pour faciliter d'éventuelles évolutions ou transformations, impossibles à loger dans les premières tranches de travaux. A Cluny, c'est d'abord la démolition de la vieille abbatiale qui est confiée aux successeurs, prudence parfaitement compréhensible. A Nantua, l'organisation du chantier du XIe siècle (Nantua III) a épargné les bâtiments claustraux hérités de Nantua II, laissant toute liberté pour l'élaboration de projets. On ignore ce qu'ils sont devenus, adaptés ou reconstruits, mais les solutions formelles adoptées au début du XIIIe siècle semblent indiquer qu'ils ont subi des améliorations notables, notamment dans les systèmes de circulation (escalier et galerie haute), améliorations entreprise à l'occasion d'une rénovation profonde de l'église (voûtes, portails, clocher). Paray-le-Monial, enfin, montre tout le parti que l'on a pu tirer de la construction d'un organe majeur: le transept. Habilement répartie en plusieurs tranches successives, sa construction a permis de mettre en place le nouveau chapitre, en attendant de détruire l'ancien. 